\title{
Infection rate and use of antibiotics in patients with rheumatoid arthritis treated with methotrexate
}

\author{
M J van der Veen, A van der Heide, A A Kruize, J W J Bijlsma
}

\begin{abstract}
Objective-To investigate prospectively the frequency and type of infections and the use of antibiotics among patients with rheumatoid arthritis (RA) on methotrexate (MTX) and patients with $R A$ without MTX.

Methods-Every three months for one year 77 patients on MTX and 151 patients without MTX were asked about infections and the use of antibiotics by means of a standardised questionnaire. Medication was checked with the pharmacist.

Results-In the MTX group there were significantly more infections and more antibiotic therapy. The relative risks for patients on MTX of infection or antibiotics use were $1.52(95 \%$ Confidence Interval (CI) $1.04-2.22)$ and $1.49 \quad(95 \% \quad$ CI $1 \cdot 04-2 \cdot 13)$, respectively. The relative risk of MTX for respiratory tract infections was $1.43(95 \%$ CI $0.96-2 \cdot 14)$ and for skin infections $2 \cdot 19(95 \%$ CI $1 \cdot 45-3 \cdot 31)$. The increased risks could only partly be explained by differences in disease severity and were not related to either duration of MTX therapy or use of prednisone. Three patients in the MTX group had herpes zoster versus one in the control group.

Conclusions-Treatment with MTX increases the rate of infection and thus the use of antibiotics but does not lead to serious complications necessitating discontinuation of the drug.
\end{abstract}

(Ann Rheum Dis 1994; 53: 224-228)

One of the concerns arising from low-dose methotrexate (MTX) therapy for rheumatoid arthritis (RA) is a possible increase in the rate of infections. Several retrospective studies on the frequency of infections in patients with RA have shown that these patients, irrespective of treatment, are not more susceptible to common infections than control patients with osteoarthritis or soft tissue rheumatism. ${ }^{12}$ MTX affects in vitro and in vivo immunological and inflammatory reactions. In-vitro assays of the direct effects of MTX are of doubtful clinical relevance. The clinically most important finding is inhibition of granulocyte function (reduction of chemotaxis, protease activity, production of leukotriene $\mathrm{B}_{4}$ and generation of superoxide radicals). ${ }^{3}$ Chemotactic defects frequently result in cutaneous infections. ${ }^{4}$ During MTX therapy no significant changes in circulating $T$-cell population and T-cell activity are found. On the basis of these findings an increase in both cutaneous infections and prolonged and recurrent infections with staphylococcus, gram-negative organisms and fungi is to be expected during MTX treatment. In a few cases opportunistic infections have been reported in patients on MTX, sometimes in combination with corticosteroids. ${ }^{5-10}$ To our knowledge no prospective studies have been performed to estimate the frequency of infection in either RA patients or MTX-treated patients with RA.

In this prospective study the occurrence of infections, especially infections of the respiratory and urinary tracts and the skin, was investigated in a group of patients with RA on MTX therapy and a group of control patients with RA on other antirheumatic drugs. At the start of the study, data on infections in the preceding year were collected; retrospective and prospective data were compared.

\section{Patients and methods}

Between 1 July 1989 and 30 November 1990, 90 patients with RA, as defined by the American College of Rheumatology 1987 revised criteria, ${ }^{11}$ who were attending the outpatient clinic of the University Hospital Utrecht, were treated with MTX and were included in the study. For every MTX-treated patient approximately two control patients with RA ( $n=152)$ who did not use MTX during the study and visited the outpatient clinic at least once every three months, were selected. At the start of the study and every three months for one year patients were questioned about the occurrence of infections and the use of antibiotics by means of a standardised questionnaire. More detailed questions covered the symptoms of infections of the urinary tract, upper respiratory tract and skin. Coughing and production of sputum were scored as respiratory infection. Selflimiting periods of general malaise with or without fever were classified as 'influenza' (flu) and sneezing and a runny nose as a 'common cold'. Skin infections were defined as draining ulcers surrounded by an indurated zone. All other infections were categorised as 
'miscellaneous'. Additional information consisted of diagnosis of the infection by a physician, with or without laboratory tests and/ or radiodiagnostic investigation. The same questionnaire was used for a retrospective inventory of infections and on the use of antibiotics during the year preceding the study. At the end of the study, after permission was granted by all patients, the reported use of antibiotics was checked with the regular pharmacist. In the Netherlands everyone depends on one pharmacy for his or her drugs. However, only one pharmacy is open at night and during the weekend. Antibiotics can only be obtained through a doctor.

Data on 228 patients were obtained from the medical records on sex, duration of $R A$, rheumatoid factor status, history of joint prostheses and cardiovascular grafts and functional class according to the criteria of Steinbrocker (scale 1-4).12 Functional capacity of the lower limbs, that is, 'mobility', was scored separately: $\mathrm{I}=$ no limitation in walking, II = limitation in walking outdoors, III = only walking indoors, $\mathrm{IV}=$ bedridden or confined to wheelchair. At all consultations the use of the following medications was registered: disease modifying antirheumatic drugs (DMARDs), nonsteroidal anti-inflammatory drugs (NSAIDs), corticosteroids and antibiotics. Blood samples were taken for deter-

Table 1 Characteristics of the patients at the start of the study

\begin{tabular}{lll}
\hline & $\begin{array}{l}\text { MTX group } \\
n=77\end{array}$ & $\begin{array}{l}\text { Control group } \\
n=151\end{array}$ \\
\hline Female (\%) & 77 & 70 \\
Age, mean (SD) & $57 \cdot 9(15)$ & $60 \cdot 1(13 \cdot 7)$ \\
(range) & $(19-81)$ & $(28-85)$ \\
Rheumatoid factor positive (\%) & 90 & 79 \\
Duration of RA (years), mean (SD) & $13 \cdot 6(10 \cdot 4)$ & $11 \cdot 5(10 \cdot 3)$ \\
(range) & $(1-44)$ & $(0 \cdot 5-51)$ \\
Functional class (\%) & & \\
$\quad$ I & 40 & 65 \\
$\quad$ II & 44 & 23 \\
$\quad$ III & 12 & 11 \\
IV & 4 & 1 \\
Mobility (\%) & & 43 \\
I & 22 & 49 \\
II & 66 & 7 \\
III & 9 & 1 \\
IV & 3 & 12 \\
Joint prostheses (\%) & 18 & 3 \\
Cardiovascular grafts (\%) & 1 & \\
\hline
\end{tabular}

*Significantly different between groups, $p<0.01$ (Chi square).

Table 2 Medication at the start of the study

\begin{tabular}{lcc}
\hline & $\begin{array}{l}\text { MTX group } \\
n=77\end{array}$ & $\begin{array}{l}\text { Control group } \\
n=151\end{array}$ \\
\hline NSAID (\%) & 91 & 90 \\
DMARD (\%) & & \\
None & & 37 \\
Hydroxychloroquine & & 12 \\
Auromyose & & 11 \\
Auranofin & & 12 \\
Sulfasalazine & & 20 \\
Azathioprine & 100 & 2 \\
d-Penicillamine & & 6 \\
Methotrexate & 88 & \\
Prednisone (\%) & 11 & 89 \\
0 & 1 & 7 \\
１0 mg/day & 0 & 3 \\
10-20 mg/day & & 1 \\
$\geqslant 20$ mg/day & & \\
\hline
\end{tabular}

mination of blood chemistry and blood counts.

\section{STATISTICS}

Statistical significance of differences in binomial or categorised variables was assessed with Chi-square tests with the Yates correction; in the event of small numbers Fisher's exact test was used. Differences in continuous variables between groups were analysed with the Mann-Whitney U test. Relative risks (RRs) for the occurrence of infections and use of antibiotics were calculated for the MTX group versus the control group; crude RRs as well as RRs stratified for disease severity (functional class) and use of prednisone were calculated. All analyses were performed with the statistical packages SPSS-PC+ and Epi-Info (RRs).

\section{Results}

PATIENTS STUDIED

Initially 90 patients were included in the MTX group. At the start of the study 54 patients had been using MTX (5-15 mg per week) longer than six months (median 19 months, range 6-63 months) and 36 for less than six months. Ten patients terminated the use of MTX during the study: 1 because of lack of efficacy and 9 because of side effects (1 nausea, 1 diarrhoea, 1 malaise, 1 hair loss, 1 nodulosis and skin ulcers, 2 psychological reasons, 2 because of pulmonary infiltrates and fever (suspicion but no proof of MTX pneumonitis)). Three patients were lost to follow up. Data on these 13 patients were not included in the calculations. The control group consisted initially of 152 patients; one patient was lost to follow up. Thus analysis covered findings on 77 patients in the MTX group and 151 patients in the control group.

None of the patients had Felty's syndrome, and neither neutropenia nor lymphopenia was found during the study.

In the MTX group functional class and mobility scores were significantly higher (that is, worse) than in the control group (table 1). No differences in other disease characteristics (rheumatoid factor positivity, disease duration) were found.

MEDICATION DURING THE STUDY

Similar percentages of the patients in both groups used NSAIDs. Of the patients in the control group $37 \%$ did not use a DMARD, most probably due to the less severe RA in this group compared with the MTX group (table 2). No difference was found between the two groups in the proportion of patients taking prednisone or the average prednisone dose. During the study no important changes in the use of NSAIDs, DMARDs or prednisone were seen.

INFECTIONS AND THE USE OF ANTIBIOTICS Table 3 shows reported infections and reported use of antibiotics for both groups. In 
Table 3 Infections and use of antibiotics during the prospective study

\begin{tabular}{lll}
\hline & $\begin{array}{l}\text { MTX group } \\
n=77\end{array}$ & $\begin{array}{l}\text { Control group } \\
n=151\end{array}$ \\
\hline Infections (\%) & $62^{\star}$ & 47 \\
Skin & $12^{\star}$ & 3 \\
Respiratory tract & 23 & 15 \\
Urinary tract & 9 & 9 \\
Sinusitis & 5 & 2 \\
Influenza & 5 & 9 \\
Cold & 4 & 8 \\
Miscellaneous & 17 & 12 \\
No infections (\%) & 38 & 53 \\
Use of antibiotics (\%) & $40^{\star}$ & 26 \\
Data pharmacist (\%) & & \\
available & 84 & 90 \\
$\quad$ concordant $\dagger$ & 72 & 85 \\
$\quad$ discordant & 28 & 15 \\
not available & 16 & 10 \\
\hline
\end{tabular}

*Significantly different from control group, $\mathrm{p}<0.05$ (Ch

square).
tConcordant, that is, patient's information agrees with tConcordant, that

pharmacist's record. antibiotics than recorded by pharmacist.

Table 4 Relative risk that patients on MTX acquired an infection during the prospective study

\begin{tabular}{lll}
\hline & $\begin{array}{l}\text { Relative risk } \\
(95 \% C I)\end{array}$ & $\begin{array}{l}\text { Stratified } \\
\text { relative risk* } \\
(95 \% C I)\end{array}$ \\
\hline Overall infections & $1 \cdot 52$ & $\begin{array}{l}1.40 \\
(0.95-2.05)\end{array}$ \\
Skin infections & $(1.04-2 \cdot 22)$ & $\begin{array}{l}2 \cdot 08 \\
(1 \cdot 41-3 \cdot 09)\end{array}$ \\
Respiratory tract infections & $\begin{array}{l}2 \cdot 19 \\
(1.45-3 \cdot 31)\end{array}$ & $\begin{array}{l}1.35 \\
(0.93-1.98)\end{array}$ \\
Use of antibiotics & $(0.96-2 \cdot 14)$ & 1.34 \\
& 1.49 & $(0.95-1 \cdot 89)$ \\
\hline
\end{tabular}

*Stratification for functional class.

table 4 the relative risks that patients on MTX will develop infections or use antibiotics are shown. A significant difference was found between the MTX group and the control group for the overall frequency of infections: $62 \%$ and $47 \%$, respectively, $R R=1.52(95 \% \mathrm{CI}$ $1 \cdot 04-2 \cdot 22)$. This difference can be explained mainly by the increased occurrence of skin infections in the MTX group: $12 \%$ versus $3 \%$, $\mathrm{RR}=2 \cdot 19 \quad(95 \%$ CI $1.45-3.31), \quad$ and respiratory tract infections: $23 \%$ versus $15 \%$, $\mathrm{RR}=1 \cdot 43(95 \%$ CI $0 \cdot 96-2 \cdot 14)$. Stratification of the RRs for functional class showed the increased risk of infections for the MTX group to be only partially due to disease severity: the stratified RR for MTX was 1.40 (95\% CI $0.95-2.05)$ for overall infections, $2.08(95 \% \mathrm{CI}$ $1.41-3.09)$ for skin infections and $1.35(95 \%$ CI $0.92-1.98$ ) for respiratory tract infections.

In the MTX group more sinusitis and miscellaneous infections were reported whereas influenza and colds were common in the control group, but these differences did not reach statistical significance. The category of miscellaneous infections consisted of herpes zoster $(n=4)$, herpes simplex $(n=5)$, varicella $(n=1)$, erysipelas $(n=3)$, infected joint prostheses $(n=3)$, vaginal infections $(n=2)$, prostatitis $(n=2)$, viral pericarditis $(n=1)$ and Candida stomatitis $(n=2)$. Three patients on MTX ( 2 in combination with prednisone) and 1 patient of the control group (using a combination of sulfasalazine and prednisone) experienced a herpes zoster infection; the other infections were distributed equally over both groups. In the MTX group 22 patients reported more than one infection versus 30 patients in the control group.

The percentage of patients reporting that they had used antibiotics was $40 \%$ in the MTX group and $26 \%$ in the control group ( $p<0.05)$, with an RR stratified for functional class of $1.34(95 \%$ CI $0.95-1.89)$ for use of antibiotics in the MTX group. We checked all medication with the pharmacists, but their records did not always go back far enough. Nevertheless, the information provided by $80 \%$ of the patients agreed with the pharmacists' records. For the remaining $20 \%$ equal proportions of patients reported taking more or less antibiotics than recorded by their pharmacist. Patient information therefore can be considered to be reliable.

Stratification for use of prednisone did not affect the RRs for infections or use of antibiotics (data not shown).

Duration of MTX therapy did not affect the occurrence of infections or the use of antibiotics either. When duration of MTX therapy at the start of the study was divided into the categories $0-2$ months $(n=24), 3-12$ months $(n=31)$ and 13-60 months $(n=22)$ infections occurred in $58 \%, 55 \%$ and $73 \%$, respectively $(p>0.05)$. Antibiotics were used by $33 \%, 42 \%$ and $41 \%$, respectively $(\mathrm{p}>0.05)$

\section{RETROSPECTIVE DATA}

Retropective data from the year preceding the study did not reveal any significant difference between the two groups in overall frequency of infections, although the percentage of reported respiratory tract infections was significantly higher in the MTX group than in the control group (table 5); RR $1 \cdot 73$ (95\% CI 1.11-2.70) and RR stratified for functional class 1.52 (95\% CI 0.99-2.33). The use of antibiotics did not differ significantly between the two groups.

Table 5 Infections and use of antibiotics during the retrospective study

\begin{tabular}{lcc}
\hline & $\begin{array}{l}\text { MTX group } \\
n=77\end{array}$ & $\begin{array}{l}\text { Control group } \\
n=151\end{array}$ \\
\hline Infections (\%) & $35 \dagger$ & $28 \dagger$ \\
Skin & 3 & 1 \\
Respiratory tract & $14^{\star}$ & $5 \dagger$ \\
Urinary tract & 10 & 7 \\
Sinusitis & 3 & 1 \\
Influenza & 0 & $3 \dagger$ \\
Cold & 1 & 3 \\
$\quad$ Miscellaneous & 13 & 11 \\
No infections (\%) & 65 & 72 \\
Use of antibiotics (\%) & 30 & 21 \\
Data pharmacist (\%) & & \\
available & 57 & 47 \\
$\quad$ concordant $\dagger \ddagger$ & 82 & 90 \\
$\quad$ discordant\$ & 18 & 10 \\
not available & 43 & 53 \\
\hline
\end{tabular}

*Significantly different from control group, $p<0.05$ (Chi square).

tSignificantly different from prospective data, $\mathrm{p}<0.05$ (Ch square) (compare with table 3 ).

$\neq$ Concordant, that is, patient's information agrees with pharmacist's record.

Discordant, that is, patient reports either more or less use of antibiotics than recorded by pharmacist. 
Comparison with the prospective data revealed that a significanly lower percentage of the patients reported infections retrospectively, in the MTX group as well as the control group. In the control group significantly fewer respiratory tract infections were reported retrospectively.

\section{Discussion}

The aim of this study was to determine whether the administration of low-dose MTX to patients with RA is associated with an increased risk of infection. Prospective selfreported patient information indicated that a significantly higher percentage of patients had had infections and used antibiotics in the MTX group than in a control group of patients with RA not on MTX therapy. The difference in overall infections was due mainly to an increased rate of skin infections and respiratory tract infections. Stratification for disease severity (functional class) only slightly reduced the relative risk for infections and use of antibiotics. Use of prednisone and duration of MTX therapy did not influence the occurrence of infections or the use of antibiotics.

In this study draining ulcers surrounded by an indurated zone were regarded as skin infections. It is not always easy to determine clinically whether skin ulcers on fingers or toes are based on infection or on vasculitis; clear vasculitis, however, was not considered as an infection in this study.

Respiratory tract infections did not lead to discontinuation of MTX therapy. In two cases MTX was stopped because of fever and pulmonary infiltrates. Pathogenic microorganisms could not be isolated and since methotrexate pneumonitis could not be ruled out, MTX was stopped. In our clinic, all patients who start MTX therapy are asked routinely about pulmonary complaints and dyspnoea; medical examination, $x$ rays and, if possible, lung function tests are performed. When one of the results is not satisfactory, MTX is not given. By excluding patients with pulmonary problems from treatment with MTX, we made a selection concerning respiratory problems which favoured the MTX group. The higher incidence of respiratory tract infections found for the MTX group cannot therefore be explained by baseline differences in pulmonary status.

In previous reports opportunistic infections have been reported in patients using MTX. In our clinic we have seen one Nocardia infection and one very serious multidermatome herpes zoster infection in RA patients on MTX. . $^{5-10}$ During this study three patients $(4 \%)$ of the MTX group experienced a mild herpes zoster infection, which did not necessitate withdrawal of MTX, versus one patient $(0.6 \%)$ of the control group. Although our numbers on this specific infection are too small to draw conclusions, this finding seems to agree with those reported by Antonelli: herpes zoster may occur more frequently in patients with RA on MTX therapy although it appears to be selflimiting and benign. ${ }^{8}$ Whereas Antonelli found a relationship with disease duration but not with the use of prednisone, the mean disease duration for patients in this study who experienced herpes zoster did not differ from that of the rest of the group $(9.5$ years, range four-14 years) and three (75\%) of them used prednisone ( 2 in the MTX group and 1 in the control group). These results seem to differ from Antonelli's, but again the numbers are too small to draw conclusions. No other opportunistic infections were seen in this patient group.

The frequency of infections was not related to the duration of treatment with MTX. From this finding it can be concluded that MTX does not have a strong immunosuppressive effect. In their long-term follow up studies of MTX therapy for RA, Weinblatt and Kremer also found no relationship between duration of therapy and clinical toxicity. ${ }^{13} 14$

In the MTX group significantly more patients reported the use of antibiotics. This may be (partly) due to a 'doctor's bias'. Doctors may be more prone to prescribe antibiotics for a patient on MTX therapy than a patient who does not take such medication. The same could apply for patients with joint prostheses, although no increase in the number of infections or use of antibiotics was found for this subgroup compared with patients without joint prostheses.

Retrospective data on our group of patients were collected for comparison with findings in previous reports. The retrospectively reported overall frequency of infections did not differ significantly between the MTX group and the control group and was comparable with findings in other retrospective studies. ${ }^{1}$ For both groups the percentage patients who reported infections was significantly higher during the prospective study than during the retrospective study, while the prospectively reported use of antibiotics did not differ significantly from the retrospective data (table 5). These findings suggest that the use of antibiotics is probably remembered more easily than the occurrence of infections. In the control group the percentage of respiratory tract infections reported during the prospective study was significantly higher than the percentage found in the retrospective data. In the MTX group this difference was not statistically significant. This may be explained by the fact that the symptoms of respiratory tract disease received more emphasis (and therefore perhaps were recalled more easily) in the MTX group because of the detailed instructions usually given to both the patients and their general practitioners at the start of MTX treatment. These results underline an important disadvantage of retrospecive studies, that is, that some data are remembered more easily than other data and that it is not advisable to rely on peoples' memory if information is to be collected adequately.

In conclusion, in our study a significantly higher overall percentage of infections was found in the MTX group, mainly due to an increased occurrence of skin infections and respiratory tract infections. Antibiotics were 
used more frequently in the MTX group than in the control group. These differences were only partly explained by differences in disease severity (functional class) and were not related to duration of MTX therapy or use of prednisone. The increase of infections did not lead to serious illness or to severe complications necessitating withdrawal of the medication.

This study was supported by a grant from The Dutch League Against Rheumatism (Het Nationaal Reumafonds).

1 van Albada-Kuipers G A, Linthorst J, Peeters E A J, Breedveld F C, et al. Frequency of infection among patients with rheumatoid arthritis versus patients with osteoarthritis or soft tissue rheumatism. Arthritis Rheum 1988; 31: 667-71.

2 Vandenbroucke J P, Kaaks R, Valkenburg H A, et al. Frequency of infections among rheumatoid arthritis Frequency of infections among rheumatoid arthritis
patients, before and after disease onset. Arthritis Rheum patients, before

3 Denman A M, Brooks P M. Antirheumatic therapy. In: Maddison P J, ed. Oxford textbook of rheumatology. Oxford: Oxford University Press, 1993: 339-40.

4 Densen P, Mandell G L. Granulocytic phagocytes. In: Mandell G L, ed. Principles and practice of infectious diseases. New York: John Wiley and Sons, 1985: 63-4.
5 Perruquet J L, Harrington Th M, Davis D E. Pneumocystis carinii pneumonia following methotrexate therapy for rheumatoid arthritis. Arthritis Rheum 1983; 26: 1291-2.

6 Altz-Smith M, Kendall L G, Stamm A M. Cryptococcosis associated with low-dose methotrexate for arthritis. $A m \mathcal{F}$ Med 1987; 83: 179-81.

7 Cornelissen J J, Bakker L J, van der Veen M J, RozenbergArska M, Bijlsma J W J. Nocardia asteroides pneumonia complicating low dose methotrexate treatment of refractory rheumatoid arthritis. Ann Rheum Dis 1991; 50: 642-4.

8 Antonelli M A S, Moreland L W, Brick J E. Herpes zoster in patients with rheumatoid arthritis treated with weekly, low-dose methotrexate. Am $\mathcal{F}$ Med 1991; 90: 295-8.

9 Aboguddah A, Stein H B, Phillips P, Amar J, English R. Herpes simplex hepatitis in a patient with psoriatic arthritis taking prednisone and methotrexate. Report and arthritis taking prednisone and methotrexate. Report
review of the literature. $₹$ Rheum $1991 ; 18: 1406-12$.

10 Clerc D, Brousse C, Mariette X, Bennet P, Bisson M. Cytomegalovirus pneumonia in a patient with rheumatoid arthritis treated with low dose methotrexate and prednisone. Ann Rheum Dis 1991; 50: 67.

11 Arnett F C, Edworthy S M, Bloch D A, et al. The American Rheumatism Association 1987 revised criteria for the classification of rheumatoid arthritis. Arthritis Rheum 1988; 31: 315-23.

12 Steinbrocker $\mathrm{O}$, Traeger $\mathrm{C} \mathrm{H}$, Batterman $\mathrm{R} C$. Therapeutic criteria in rheumatoid arthritis. $\mathcal{F} A M A$ 1949; 140: criteria

13 Weinblatt M E, Weissman B N, Holdsworth D E, et al. Long-term prospective study of methotrexate in the treatment of rheumatoid arthritis. Arthritis Rheum 1992; 35: 129-37.

14 Kremer J M, Phelps C T. Long-term prospective study of the use of methotrexate in the treatment of rheumatoid arthritis. Arthritis Rheum 1992; 35: 138-45. 